\title{
ESTADO NUTRICIONAL E NÍVEIS PRESSÓRICOS DE ESCOLARES ADOLESCENTES DA CIDADE DE CUBATÃO - SP, BRASIL.
}

\section{NUTRITIONAL STATUS AND BLOOD PRESSURE LEVEL OF ADOLESCENTS OF CUBATÃO CITY, SP - BRAZIL}

Márcia Cristina Pires da Silva ${ }^{*+}$

Cecília Helena Bicalho Ramos **

Roberto Fernandes da Costa ${ }^{*+}$

Silva MCP. da Ramos CHB. Costa RF. da. Estado Nutricional e Níveis Pressóricos de Adolescentes da Cidade de Cubatão - SP, Brasil. Rev Bras Crescimento Desenvolvimento Hum. 2008; 18(3): 288-297

\section{Resumo:}

O aumento da prevalência de obesidade em adolescentes tem contribuído para o aparecimento precoce da hipertensão arterial. Objetivos: Verificar o estado nutricional e os níveis pressóricos de adolescentes, bem como a relação entre estes fatores e diferenças entre os sexos. Método: Estudo descritivo com delineamento transversal realizado em duas escolas públicas da cidade de Cubatão. Participaram 704 adolescentes de 10 a 15 anos de idade, sendo 333 rapazes e 371 moças. Para avaliação do estado nutricional foi utilizado o índice de massa corporal por idade e sexo. A pressão arterial foi medida por meio da técnica auscultatória e os valores encontrados foram avaliados de acordo com o percentil de estatura para a idade dos adolescentes. O tratamento estatístico constou da apresentação da proporção dos sujeitos em sobrepeso e obesidade, bem como dos que apresentavam alterações na pressão arterial. Foi utilizado o teste Qui-quadrado para verificar diferença de proporção de alterações pressóricas entre sujeitos eutróficos e com sobrepeso ou obesidade, além do cálculo de Odds Ratio e IC95\%. A significância estatística adotada foi de p < 0,05. Resultados: A avaliação do estado nutricional mostrou que 13,64\% dos adolescentes apresentavam sobrepeso e 10,37\% eram obesos. Quanto aos níveis pressóricos, 12,65\% dos adolescentes apresentaram pressão Normal Alta e 9,52\% apresentaram Hipertensão. Os adolescentes com excesso de peso apresentaram significativamente maior proporção de alterações pressóricas: $\chi^{2}=15,39(\mathrm{p}=0,00008)$; OR = 2,23 (IC\% = 1,47-3,37). Conclusão: Os resultados mostraram elevadas proporções de excesso de peso e alterações pressóricas, com clara relação entre eles.

Palavras-chave: Hipertensão; sobrepeso; obesidade; adolescentes; estado nutricional; escolares.

Apresentado no $62^{\circ}$ Congresso Brasileiro de Cardiologia, São Paulo em 17/10/2007.

*+ $\quad$ CEPS - Centro de Estudos e Pesquisas Sanny - Rua: Brasil, 791/795 - SBC - São Paulo - CEP: 09627-000

*+ Grupo de Pesquisa em Disfunção do Movimento Humano da Faculdade de Fisioterapia da Universidade Santa Cecília.

** Prefeitura Municipal de Cubatão.

Correspondência para: Roberto Fernandes da Costa. Av Afonso Penna, nº167 - cj 91- Santos/São Paulo - CEP: 11020001. Tel:(13)3301 0630.Fax: (13) 3301 0640. E-mail: roberto.costa@cepsanny.com.br 


\begin{abstract}
:
The increase in obesity prevalence in adolescents has been contributing to earlier hypertension. Objective: To verify nutritional status and blood pressure levels in adolescents, and the relationship among these factors. Methods: Descriptive cross sectional study conducted in two public schools at Cubatão city, Brazil. 704 adolescents between 10 and 15 years old, 333 boys and 371 girls participated in this study. Nutritional status assessment was taken by body mass index for age and gender. Blood pressure was measured by auscultation technique, and the values obtained were assessed in accordance to height and gender percentile of the adolescents. Statistical analysis was made by proportion of subjects with overweight or obesity, and with blood pressure alterations. Chi-square test was used to verify proportion differences of blood pressure alterations between normal weight and overweight subjects, as well as Odds Ratio and 95\% CI. It was adopted statistic significance to $\mathrm{p}<0.05$. Results: Nutritional status assessment showed that $13.64 \%$ of adolescents were overweight and $10.37 \%$ were obese. About blood pressure alterations, $12.65 \%$ of adolescents presented High Normal blood pressure and 9.52\% Hypertension. Overweight adolescents presented significantly high proportion of blood pressure alterations: $\chi^{2}=15.39$ ( $\left.\mathrm{p}=0.00008\right)$; $\mathrm{OR}=2.23(\mathrm{CI} \%=1.47-$ 3.37). Conclusion: The results showed high proportion of overweight and blood pressure alterations, and relation among these variables.
\end{abstract}

Key words: Hypertension; overweight; obesity; adolescents; nutritional status; schoolchildren.

\section{INTRODUÇÃO}

O controle da pressão arterial tem se mostrado, cada vez mais, uma preocupação da contemporaneidade. Os hábitos de vida modernos incluindo alimentação inadequada, sedentarismo, etilismo, tabagismo e estresse têm contribuído substancialmente para o aumento da massa corporal e alterações metabólicas como dislipidemia, diabetes tipo 2 e hipertensão arterial. ${ }^{1}$

A obesidade como um problema de saúde pública mundial, tem atingido não somente adultos, mas também crianças e adolescentes em diferentes classes sociais. ${ }^{2} \mathrm{~A}$ hipertensão arterial (HA) cresceu na última década entre crianças e adolescentes, fato que apresenta entre suas causas o aumento da prevalência da obesidade nessas faixas etárias. ${ }^{3,4}$

Estudo longitudinal realizado por Sun et al. ${ }^{5}$ mostrou que o risco aumentado para hipertensão arterial e síndrome metabólica na fase adulta pode ser detectado já na primeira déca- da de vida, monitorando-se a pressão arterial de crianças a partir dos cinco anos de idade segundo esses autores, para permanecerem livres da hipertensão ou da síndrome metabólica as crianças devem apresentar níveis pressóricos, abaixo do percentil 50 para idade e sexo. ${ }^{5}$

O excesso de gordura corporal independente de sua distribuição apresenta-se como um indicativo de risco para o desenvolvimento de doenças cardiovasculares. ${ }^{6} \mathrm{O}$ infarto agudo do miocárdio pode ocorrer em adolescentes que apresentem uma anatomia coronariana normal. $\mathrm{E}$ as implicações deste em eventos cardíacos futuros, nas suas atividades e estilo de vida, bem como seu tratamento em longo prazo ainda são desconhecidas. Assim, identificar e estabelecer um acompanhamento até a idade adulta destes jovens assume fundamental importância. ${ }^{7}$

Os benefícios da perda de peso por meio de exercícios e atividade física para a redução da pressão sanguínea em crianças foram de- 
monstrados e sugeridos em estudos observacionais e de intervenção..$^{8,9}$

O excesso de peso está diretamente associado à presença de HA já na infânciaa,13, portanto, o esclarecimento dos profissionais da saúde, dos familiares e educadores é fundamental para que a prevenção e o tratamento da obesidade sejam alcançados com êxito. ${ }^{9}$

A HA infantil é uma patologia com alta morbidade, além de ser preditora de HA na vida adulta, assim tanto a prevenção quanto o diagnóstico precoce, nas primeiras etapas da vida, são essenciais. Tal fato justifica a necessidade de estudos que indiquem a presença de risco para a saúde decorrentes do excesso de gordura corporal e de alterações pressóricas, em período precoce, como a idade escolar.

Considerando que o aumento da obesidade e da hipertensão arterial na infância têm sido demonstrados em diferentes classes sócioeconômicas ${ }^{2,4}$, em diferentes regiões, possivelmente tal fato seja confirmado em escolares da cidade de Cubatão.

Neste contexto, o objetivo é verificar o estado nutricional e os níveis pressóricos de adolescentes da cidade de Cubatão, bem como a relação entre estes fatores, além das possíveis diferenças entre os sexos.

\section{MÉTODO}

A cidade de Cubatão tem área total de Cubatão é de 142,3 $\mathrm{Km}^{2}$ de extensão. Cubatão tem seu clima quente e úmido, com sua temperatura bastante variável. A umidade relativa do ar é elevada superior a $80 \%$ em certas épocas do ano. $\mathrm{Na}$ área fisiográfica da Baixada Santista, Latitude 2352'26"82 e Longitude 46²5’37"08. É localizada a 57 km de distância da Capital do Estado de São Paulo. A população de Cubatão conforme dados estimativos do IBGE de 2006 é de 121.002 habitantes aproximadamente. (FONTE: www.ibge.gov.br)
Trata-se de um estudo descritivo com delineamento transversal. A amostra intencional foi constituída de todos os alunos matriculados em duas escolas da rede municipal de ensino fundamental ( $5^{\mathrm{a}}$ a $8^{\mathrm{a}}$ séries), da cidade de Cubatão (SP), com idades de 10 a 15 anos. Para compor a amostra foram selecionados alunos de todas as séries das duas maiores escolas da rede municipal da cidade por estarem localizadas no centro e permitirem fácil acesso. Participaram do estudo 704 alunos autorizados pelos pais por meio de termo de consentimento livre e esclarecido, sendo 333 do sexo masculino e 371 do sexo feminino; após registradas 17 perdas, sendo duas por falta, uma por recusa e 14 porque se encontravam fora da faixa etária estipulada.

As medidas antropométricas de massa corporal e estatura foram realizadas utilizando-se padronização descrita na literatura, estando os adolescentes com roupas leves e descalços, para a avaliação com balança portátil digital da marca CAMRY ${ }^{\circledR}$ - modelo BMI, e estadiômetro portátil da marca Sanny ${ }^{\circledR}$ - modelo Personal.

O IMC foi calculado dividindo-se a massa pela estatura ao quadrado $\left(\mathrm{kg} / \mathrm{m}^{2}\right)$. A classificação do estado nutricional foi realizada de acordo com os critérios propostos pelo CDC $^{14}$ : baixo peso, IMC inferior ao percentil 5; eutrofia, IMC igual ou superior ao percentil 5 e inferior ao percentil 85; sobrepeso, IMC igual ou superior ao percentil 85 e inferior ao percentil 95; obesidade, IMC igual ou superior ao percentil 95.

Para a medida da pressão arterial, utilizou-se de técnica padronizada com equipamento calibrado pelo INMETRO. A técnica auscultatória foi aplicada com esfigmomanômetro aneróide da marca Welch Allyn Tycos ${ }^{\circledR}$ e estetoscópio da marca Littmann ${ }^{\circledR}$. A medida da pressão arterial foi realizada de acordo com o procedimento descrito nas V Diretrizes Brasileiras de Hiperten- 
são. ${ }^{15}$ Assim, os valores encontrados foram avaliados de acordo com o percentil de estatura para a idade dos adolescentes.

Foram considerados hipertensos os sujeitos que apresentaram pressão arterial sistêmica em nível igual ou superior ao valor correspondente ao percentil 90 da tabela de pressão arterial referente ao sexo e ao percentil de estatura para cada faixa de idade. ${ }^{15}$

Todas as medidas foram realizadas durante o período em que os alunos estavam na escola, sendo aproximadamente metade pela manhã, entre $08 \mathrm{~h} 00$ e $12 \mathrm{~h} 00$; e metade à tarde, entre $13 \mathrm{~h} 00$ e $17 \mathrm{~h} 00$.

O tratamento estatístico constou da apresentação da proporção dos sujeitos em sobrepeso e obesidade, bem como dos que apresentavam alterações na pressão arterial. Foi utilizado o teste Qui-quadrado para verificar diferença de proporção entre os sexos, e de alterações pressóricas entre sujeitos eutróficos e com sobrepeso ou obesidade, além do cálculo de Odds Ratio e IC95\%. A significância estatística adotada foi de $\mathrm{p}<0,05$. As análises foram realizadas com auxílio do software SPSS-v11.0.
A realização deste estudo obedeceu aos princípios éticos para pesquisa envolvendo seres humanos, conforme resolução CNS 196/96, após aprovação pelo Comitê de Ética em Pesquisa da Universidade Santa Cecília sob número 07/2007.

\section{RESULTADOS}

A avaliação do estado nutricional mostrou que $10,37 \%$ dos adolescentes avaliados apresentavam obesidade, 13,64\% sobrepeso, $71,16 \%$ eram eutróficos e $4,83 \%$ se encontravam em baixo peso. Na comparação entre os sexos não houve diferença estatisticamente significante quanto ao aspecto nutricional (Tabela1).

Os resultados pressóricos obtidos mostraram que $12,65 \%$ dos adolescentes apresentavam pressão arterial normal alta e 9,52\% hipertensão arterial. Na combinação das pressões sistólica e diastólica as maiores frequências observadas foram: pressão arterial normal alta sistólica associada à pressão normal diastólica

Tabela 1: Estado nutricional de adolescentes de Cubatão - SP

\begin{tabular}{cccccc}
\hline & \multicolumn{2}{c}{ Masculino } & \multicolumn{3}{c}{ Feminino } \\
\hline BP & $\mathrm{n}$ & $\%$ & $\mathrm{n}$ & $\%$ & $\mathrm{p}$ \\
$\mathrm{E}$ & 12 & 3,6 & 22 & 5,93 & 0,752 \\
$\mathrm{SP}$ & 227 & 68,17 & 274 & 73,85 & 0,206 \\
$\mathrm{O}$ & 55 & 16,52 & 41 & 11,05 & 0,435 \\
\hline
\end{tabular}

Legenda: BP: baixo peso; E: eutrófico; SP: sobrepeso; O: obesidade.

Tabela 2: Pressão arterial sistólica em adolescentes de Cubatão - SP.

\begin{tabular}{cccccccc}
\hline & \multicolumn{2}{c}{ Masculino } & \multicolumn{2}{c}{ Feminino } & \multicolumn{2}{c}{ Total } \\
\hline & $\mathrm{n}$ & $\%$ & $\mathrm{n}$ & $\%$ & $\mathrm{n}$ & $\%$ & $\mathrm{p}$ \\
\hline NA & 30 & 9,01 & 46 & 12,40 & 76 & 10,80 & 0,144 \\
$\mathrm{H}$ & 31 & 9,31 & 33 & 8,89 & 64 & 9,09 & 0,847 \\
\hline
\end{tabular}

Legenda: NA Normal Alta; H: hipertenso. 
Tabela 3: Pressão arterial diastólica em adolescentes de Cubatão - SP

\begin{tabular}{cccccccc}
\hline & \multicolumn{2}{c}{ Masculino } & \multicolumn{2}{c}{ Feminino } & \multicolumn{3}{c}{ Total } \\
\hline & $\mathbf{n}$ & $\mathbf{\%}$ & $\mathbf{n}$ & $\mathbf{\%}$ & $\mathbf{n}$ & $\mathbf{\%}$ & $\mathbf{p}$ \\
\hline NA & 3 & 0,90 & 10 & 2,70 & 13 & 1,85 & 0,068 \\
H & 1 & 0,30 & 2 & 0,54 & 3 & 0,43 & 0,620 \\
\hline
\end{tabular}

Legenda: NA Normal Alta; H: hipertenso.

Tabela 4: Alterações pressóricas em relação ao estado nutricional

\begin{tabular}{lccc}
\hline & Total & Sujeitos com alterações pressóricas & \% \\
\hline Sem excesso de peso & 535 & 91 & 17,01 \\
Com excesso de peso & 169 & 53 & 31,36 \\
\hline
\end{tabular}

com 10,08\%, seguida de hipertensão sistólica associada à normotensão diastólica com 8,10\% do total. Não houve diferença estatisticamente significante entre as alterações pressóricas quanto ao sexo (Tabelas 2 e 3 ).

Na análise da associação entre estado nutricional e níveis pressóricos dos avaliados observou-se que os adolescentes com excesso de peso apresentaram significantemente maior proporção de alterações pressóricas com $\chi^{2}=15,39(\mathrm{p}=0,00008)$ e $\mathrm{OR}=2,23$ (IC95\% = 1,47-3,37) (Tabela 4).

\section{DISCUSSÃO}

Embora o presente estudo não tenha utilizado uma amostra representativa da população de escolares adolescentes da cidade de Cubatão, o que pode constituir uma limitação, os achados obtidos são relacionados à maioria dos alunos das duas maiores escolas municipais. Assim, refletem a importância clínica do excesso de peso e das alterações pressóricas no grupo estudado.

A relação entre obesidade e hipertensão tem sido amplamente estudada. Em indivíduos obesos, alguns fatores que podem produzir alterações nas curvas da função renal são: a resistência à insulina, mudanças estruturais, alterações da função e estrutura vasculares, ativação do sistema renina-angiotensinaaldosterona, ativação do sistema nervoso autônomo simpático e do sistema hipotálamohipófise-adrenal. E, dessa forma, a habilidade dos rins em excretar sódio é alterada, causando a hipertensão arterial. ${ }^{13}$

Devido também às modificações no estilo de vida ${ }^{1,16,18}$, o adolescente, atualmente, mudou sua condição de um sujeito saudável para o que apresenta excesso de peso corporal e fatores associados, como hipertensão, histórico familiar e uma predisposição étnica para a doença hipertensiva. ${ }^{8}$

Em relação ao sexo, em um estudo realizado no Rio de Janeiro, com 456 estudantes de 12 a 17 anos de idade, entre 2003 e 2004, o sexo masculino apresentou com maior frequência pré-hipertensão em relação ao sexo feminino e além disso, a chance de prevalência de pré-hipertensão dos meninos, quando ajustada por idade, obesidade e equivalentes metabólicos, foi cerca de nove vezes maior que a das meninas. ${ }^{19}$ Em concordância, entre 5.102 
escolares americanos com média de 13,5 + 1,7 anos de idade, também houve maior prevalência dos meninos apresentarem hipertensão, no estudo de Sorof et $\mathrm{al}^{20}$, no qual encontraram um risco relativo de 1,5 entre hipertensão e sexo, com maior risco para os meninos.

Opostamente, estudo realizado em uma província de Milão, com 1.206 meninos e 1.210 meninas de escolas públicas, entre seis e 11 anos de idade, apresentou maior frequência de pressão arterial elevada nas meninas do que meninos. ${ }^{21} \mathrm{Na}$ amostra de sujeitos do presente estudo não houve diferenças estatisticamente significantes em relação ao sexo.

Estudo transversal randomizado realizado em escolas da rede pública e da rede privada de Maceió/AL, verificou sobrepeso em $9,3 \%$ dos avaliados e obesidade em $4,5 \%$, de uma amostra de 1253 indivíduos. ${ }^{11} \mathrm{Em}$ 2001, em Feira de Santana/BA, 9,1\% das 701 crianças avaliadas estavam em sobrepeso e 4,3\% eram obesas. ${ }^{9}$

Em Belo Horizonte, no mesmo ano, das 672 crianças avaliadas em uma escola pública e uma particular, 14,0\% apresentavam sobrepeso e $3,7 \%$ obesidade. ${ }^{4}$ Esses valores diferem dos encontrados no presente estudo, sendo que nestes, a proporção de obesos foi menor que a encontrada entre os adolescentes de Cubatão (10,37\% de obesidade e $13,64 \%$ de sobrepeso).

Discrepâncias como estas têm sido relatadas quando são comparados resultados obtidos em diferentes regiões do país, mesmo quando há semelhança no nível sócio-econômico. ${ }^{2}$ Uma possível explicação é a utilização de diferentes critérios para avaliação do estado nutricional, pois o critério proposto pela IOTF - International Obesity Task Force, utilizado nos estudos de Feira de Santana ${ }^{9}$ e Belo Horizonte $^{4}$, tende a subestimar a prevalência de obesidade, quando comparado ao critério do CDC, utilizado no presente estudo. ${ }^{22}$
Já na Cidade do México, também durante o primeiro semestre de 2004, em uma amostra de 561 crianças e adolescentes de nove a 12 anos, $1,1 \%$ apresentavam baixo peso, 22,7\% sobrepeso e $21,4 \%$ eram obesas. ${ }^{23}$

Quanto ao aspecto pressórico, a pressão arterial elevada avaliada isoladamente apresentou-se em 9,41\% dos 1.253 estudantes de sete a 17 anos, e, quando considerada a média das duas medidas a prevalência foi de 7,7\% no estudo de Moura. ${ }^{11}$

Já no de Perichart-Pereira ${ }^{23}, 8,4 \%$ das 561 crianças tinham pressão arterial sistólica elevada e 3,8\% pressão arterial diastólica elevada não havendo diferença estatisticamente significante entre os sexos. Das 3.169 crianças de sete a 14 anos, avaliadas por Monego ${ }^{16}$, 5\% eram hipertensas e 6,2\% apresentavam pressão normal alta.

Valores ainda mais elevados foram os encontrados em um estudo realizado no Texas, em Fort Worth, com 1.018 crianças, no qual a prevalência de hipertensão foi de 20,6\%. ${ }^{17} \mathrm{Em}$ Houston, de 2.460 estudantes de 12 a 16 anos de idade de oito escolas da rede pública, 16,8\% eram hipertensos e $24,1 \%$, apresentaram pressão normal alta. $^{8}$ Em outro estudo de corte transversal realizado de 2003 a 2005, dos 6.790 adolescentes de 11 a 17 anos avaliados, 9,4\% apresentavam hipertensão e 9,5\% pré-hipertensão. ${ }^{24}$ No presente estudo, verificou-se que $12,65 \%$ dos adolescentes apresentavam pressão normal alta e 9,52\% hipertensão.

Essa ampla variação entre os achados epidemiológicos na literatura a respeito da prevalência de hipertensão arterial em escolares ainda mostra valores bastante discrepantes, o que pode decorrer da utilização de métodos inadequados que conduzem a superestimação da taxa de crianças hipertensas. ${ }^{9}$ Porém, devese também a diversos aspectos, tanto biológicos e genéticos como a etnia ${ }^{17,20}$, sexo, faixa etária e aspectos hormonais; quanto a aspectos econômicos e sociais, como hábitos de 
vida $^{1,16,18}$, os quais são diretamente influenciados pela cultura e renda familiar. Dessa forma, para a prevenção e o tratamento de distúrbios pressóricos e nutricionais, todos esses aspectos devem ser levados em consideração.

Ao se associar os níveis pressóricos com o estado nutricional dos estudantes de Cubatão, verificou-se que os indivíduos com excesso de peso apresentavam maiores proporções de alterações pressóricas $(\mathrm{OR}=2,23$; IC95\% = 1,47 - 3,37), em concordância com outros estudos nos quais a pressão elevada esteve presente quando coexistia excesso de peso corporal. ${ }^{4,6,9-11,21,25,26}$

A resistência à insulina tem sido sugerida, assim como a obesidade abdominal, como fator etiológico em potencial para a doença cardiovascular. ${ }^{27}$ Além disso, estudos afirmam que o risco de hipertensão tardia depende mais da taxa de crescimento desde o nascimento que do tamanho do corpo alcançado em toda vida adulta $^{28}$, e na infância estes fatores são determinantes da pressão sanguínea na vida adulta. ${ }^{29}$ Dessa forma, a combinação de dieta e atividade física visando à redução de peso $^{10}$, já na infância, mostra-se fundamental na prevenção e tratamento da síndrome metabólica. ${ }^{25,27}$

Esta relação entre obesidade e hipertensão tem grande importância clínica, uma vez que a redução de peso mesmo modesta (da ordem de 5-10\% do peso inicial), mas, mantida em longo prazo (3-4 anos), tem como consequência a redução da pressão arterial diastólica em 0,35 mmHg e da sistólica em 0,45 mmHg para cada quilograma de peso perdido. ${ }^{30}$

O tratamento farmacológico da obesidade em adultos é repleto de problemas, sendo poucos os estudos controlados que mostram os efeitos e eficácia para o seu uso na obesidade infantil. ${ }^{8}$ Assim, apesar das dificuldades que possam existir em se identificar os fatores de risco da obesidade e suas complicações em crianças, a prevenção é a melhor estratégia. ${ }^{8}$ Além disso, a perda de peso em conjunto com exercícios físicos pode ser muito benéfica no combate à obesidade e hipertensão infantil, entre outras doenças. , $^{8,17,31}$

De acordo com Jago et al. ${ }^{31}$, a estratégia na prevenção da obesidade antes da puberdade deve enfocar a promoção da atividade física e diminuição no tempo de atividades sedentárias, e consequentemente, aumento no gasto energético, visto que em seus achados o tempo dedicado à assistência à TV e atividade física foram os únicos que interferiram nos índices da massa corporal. O que pode ser explicado pelo fato da variabilidade da pressão sanguínea ser maior durante o período diurno e apresentar associação com o aumento rápido da glicemia em crianças hipertensas ${ }^{32}$, demonstrando assim que a atividade física é uma importante aliada no combate à obesidade ${ }^{25}$ e à hipertensão. ${ }^{12,31,32}$

A identificação precoce desses fatores de risco, a modificação do estilo de vida ${ }^{1,}$ 16, 18, incluindo o controle do peso corporal, realização de exercícios ${ }^{24}$ de forma regular, melhora da qualidade da alimentação por meio de orientação dietética adequada ${ }^{6}$, além da monitorização rotineira da pressão arterial $^{18,34}$ e estado nutricional das crianças, constituem medidas preventivas fundamentais contra futuros eventos cardiovasculares. $^{4,20}$

A utilização do IMC por idade e sexo, com o percentil 85 como valor de corte para sobrepeso e 95 para obesidade, pressupõe que crianças e adolescentes apresentem no máximo 10\% de prevalência de sobrepeso e $5 \%$ de obesidade ${ }^{14}$, sendo que no presente estudo foi encontrado valores superiores a estes em mais de 30,0\% para o sobrepeso e mais de $100 \%$ para a obesidade. 
Nos estudos realizados no Brasil a prevalência de hipertensão na adolescência tem variado de $2,0 \%$ a $11,0 \%{ }^{26}$, assim, os resultados encontrados no presente estudo demonstram que os adolescentes avaliados apresentaram elevada proporção de hipertensos.

A partir de estudos como este, dada à importância do diagnóstico precoce e da integração multiprofissional no sentido de prevenção e tratamento da obesidade e da hipertensão infantil, devem ser propostos programas que envolvam educação, atividade física e acompanhamento nutricional para a promoção de saúde e melhora da qualidade de vida desses futuros adultos.

\section{REFERÊNCIAS}

1. Winnick M, Somers VK, Dorigatti F, Longo D, Santonastaso M, Mos L, Mattarei M, Pessina AC, Palatini P. Lifestyle, family history and progression of hypertension. J Hypertens 2006; 24 (8):1479-87.

2. Costa RF, Cintra, IP, Fisberg M. Prevalência de Sobrepeso e Obesidade em Escolares da Cidade de Santos, SP. Arq Bras Endocrinol Metab 2006; 50(1):60-67.

3. Muntner P, He J, Cutler JA, Wildman RP, Whelton PK. Trends in blood pressure among children and adolescents. JAMA 2004; 291 (17):2107-13.

4. Garcia FD, Terra AF, Queiroz AM, Correia CA, Ramos OS, Ferreira QT, Rocha RL, Oliveira EA. Avaliação de fatores de risco associados com elevação da pressão arterial em crianças. J Pediatr (RJ). 2004; 80 (1):29-34.
Assim, observam-se elevadas proporções de excesso de peso e alterações pressóricas entre os adolescentes da cidade de Cubatão, sendo que os adolescentes que apresentavam excesso de gordura corporal demonstraram maior chance de presença de hipertensão arterial.

\section{AGRADECIMENTOS}

Os autores agradecem à equipe que participou de todo o processo de coleta dos dados por sua dedicação e suporte; aos funcionários das escolas; e, principalmente, a todos os pais por autorizarem a participação de seus filhos neste estudo.

5. Sun SS, Grave GD, Siervogel RM, Pickoff AA, Arslanian SS, Daniels SR. Systolic Blood Pressure in Childhood Predicts Hypertension and Metabolic Syndrome Later in Life. Pediatrics 2007; 119:237-246.

6. Boaventura GA, Guandalini VR. Alim. Nutr 2007; 18(4):381-385.

7. Lane JR, Ben-Shachar G. MyocardialInfarction in Healthy Adolescents. Pediatrics 2007; 120(4):e938-e943.

8. Sorof J, Daniels S. Obesity hipertension in children: a problem of epidemic proportions. Hypertension 2002; 40:441-7

9. Oliveira AMA, Oliveira AC, Almeida, MS, Almeida FS, Ferreira JBC, Silva CEP, Adan LF. Fatores Ambientais e Antropométricos Associados à Hipertensão Arterial Infantil. Arq Brás Endocrinol Metabol 2004; 48(6):829-54. 
10. Mendes MJFL, Alves JGB, Alves AV, Siqueira PP, Freire EFC. Associação de fatores de risco para doenças cardiovasculares em adolescentes e seus pais. Rev Bras Saúde Matern Infant 2006; 6(Supl 1):S49-S54.

11. Moura AA, Silva MAM, Ferraz MRMT, Rivera IR. Prevalência de pressão arterial elevada em escolares e adolescentes de Maceió. J Pediatr 2004; 80(1):35-40.

12. Silva K, Lopes AS. Excesso de peso, pressão arterial e atividade física no deslocamento à escola. Arq Bras Cardiol 2008; 91(2):93-101.

13. Lima SG, Hatagima A, Silva NLCL. Sistema renina-angiotensina: é possível identificar genes de suscetibilidade à hipertensão? Arq Bras Cardiol 2007; 89(6):427-33.

14. CDC - Center for Disease Control and Prevention. 2000 CDC growth charts: United States [online] Hyaltsville: 2002. Acessado em 15 de janeiro de 2007. isponível em: <http://www.cdc.gov/ growthcharts>.

15. Brandão A, Machado CA, Amodeo C, Mion Junior D, Nobre F et al. V Diretrizes Brasileira de Hipertensão [on line] 2006 [Acesso em: $29 \mathrm{dez}$ 2008]; Disponível em: <http://www.sbn.org.br/ Diretrizes/V Diretrizes Brasileiras de Hipertensao Arterial.pdf>

16. Monego ET, Jardim PCBV.

Determinantes de risco para doenças cardiovasculares em escolares. Arq Bras Cardiol 2006; 87 (1):37-45.

17. Urrutia-Rojas X, Egbuchunam CU, Bae S, Menchaca J, Bayona M, Rivers PA, Singh KP. High blood pressure in school children: prevalence and risk factors. BMC Pediatrics 2006, 6:32.
18. Bagga A, Jain R, Vijayakumar M, Kanitkar M, Ali U. Evaluation and management of hypertension. Indian Pediatrics 2007; 44:103-21

19. Rosa MLG, Fonseca VM, Oigman G, Mesquita ET. Pré-hipertensão arterial e pressão de pulso aumentada em adolescentes: prevalência e fatores associados. Arq Bras Cardiol 2006; 87(1):46-53.

20. Sorof JM, Lai D, Turner J, Poffenbarger T, Portman RJ. Overweight, ethnicity, and the prevalence of hypertension in school-aged children. Pediatrics 2004; 113:475-82.

21. Genovesi S, Giussani M, Pieruzzi F, Vigorita F, Arcovio C, Cavuto S, Stella A. Results of blood pressure screening in a population of school-aged children in the province of Milan: role of owerweight. J Hypertensi 2005; 23:493-97.

22. Zimmermann MB, Gübeli C, Püntener C, Molinari L. Detection of overweight and obesity in a national sample of 6-12-y-old Swiss children: accuracy and validity of reference values for body mass index from the US Centers for Disease Control and Prevention and the International Obesity Task Force. Am J Clin Nutr 2004; 79:838-43.

23. Perichart-Perera O, Balas-Nakash M, Schiffman-Selechnik E, Barabato-Dosal A, Vadillo-Ortega F. Obesity increases metabolic syndrome risk factors in school-aged children from an urban school in Mexico City. J Am Diet Assoc 2007; 107:81-91.

24. Mcniece KL, Poffenbarger TS, Turner JL, Franco KD, Sorof JM, Portman RJ. Prevalence of hypertension and prehypertension among adolescents. J Pediatr 2007; 150(6):640-4. 
25. Ribeiro MM, Silva AG, Santos NS, Guazzelle I, Matos LNJ, Trombetta IC, Halpern A, Negão CE, Villares AMF. Diet and exercise training restore blood pressure and vasodilatory responses during physiological maneuvers in obese children. Circulation 2005; 111:1915-23.

26. Nogueira PK, Costa RF, Santanna J, Fisberg M, Silvestrini L. Pressão arterial elevada em escolares - relação com a obesidade. Rev Assoc Med Bras 2007; 53 (5):426-32.

27. Robinson LE, Graham TE. Metabolic syndrome, a cardiovascular disease risk factor: role of adipocytokines and impact of diet and physical activity. Can J Appl Physiol 2004; 29(6):808-29.

28. Eriksson JG, Forsén TJ, Kajantie E, Osmond C, Barker DJP. Childhood growth and hypertension in later life. Hypertension 2007; 49:1415-21.

29. Lawlor DA, Smith GD. Early life determinants of adult blood pressure. Curr Op Nephrol Hypertensi 2005; 14:259-64.
30. Stevens VJ, Obarzanek E, Cook NR, Lee I-M, Appel LJ, West DS et al, Long-term weight loss and changes in blood pressure: results of the trials of hypertension prevention, phase II. Ann Intern Med 2001; 134 (1):1-11.

31. Jago R, Baranowski T, Baranowski JC, Thompson D, Greaves KA. BMI from 3-6 $\mathrm{y}$ of age is predicted by TV viewing and physical activity, not diet. Int J Obes Relat Metab Disord. 2005; 29 (6):557-64.

32. Krzych L J. Blood pressure variability in children with essential hypertension. J Hum Hypertens 2007; 21(6):494-500.

33. Matsudo VKR, Matsudo SMM. Atividade física no tratamento da obesidade. Einstein 2006; Supl 1:S29S43.34. Lurbe E, Sorof JM, Daniels S. Clinical and research aspects of ambulatory blood pressure monitoring in children. J Pediatr 2004; 144:7-16.

Recebido em: 26/08/2008 Modificado em: 22/09/2008 Aceito em: 16/11/2008 